\title{
INTEGRAL and XMM-Newton observations towards the unidentified MeV source GRO J1411-64
}

\author{
D. F. Torres ${ }^{1}$, S. Zhang ${ }^{2}$, O. Reimer ${ }^{3}$, X. Barcons ${ }^{4}$, A. Corral ${ }^{4}$, V. Bosch-Ramon ${ }^{5}$, J. M. Paredes ${ }^{5}$, G. E. Romero ${ }^{6}$, \\ J.-L. Qu ${ }^{2}$, W. Collmar ${ }^{7}$, V. Schönfelder ${ }^{7}$, and Y. Butt ${ }^{8}$ \\ ${ }^{1}$ Institut de Ciències de l'Espai (IEEC/CSIC), Campus UAB, Facultat de Ciències, Torre C5-parell, $2^{\text {da }}$ planta, \\ 08193 Barcelona, Spain \\ e-mail: dtorres@ieec.uab.es \\ ${ }^{2}$ Laboratory for Particle Astrophysics, Institute of High Energy Physics, Beijing 100049, China \\ 3 W. W. Hansen Experimental Physics Laboratory, Stanford University, Stanford, CA 94305, USA \\ 4 Instituto de Física de Cantabria (CSIC-UC), 39005 Santander, Spain \\ 5 Universitat de Barcelona, Av. Diagonal 647, 08028 Barcelona, Spain \\ 6 Instituto Argentino de Radioastronomia, CC5, 1894 Villa Elisa, Argentina \\ 7 Max-Planck-Institut für extraterrestrische Physik, PO Box 1603, 85740 Garching, Germany \\ 8 Harvard-Smithsonian Center for Astrophysics, 60 Garden St., Cambridge, MA 02138, USA
}

Received 3 April 2006 / Accepted 16 June 2006

\section{ABSTRACT}

\begin{abstract}
Aims. We investigate the nature of the COMPTEL unidentified source GRO J1411-64.
Methods. The source was observed by INTEGRAL. Its central part was also observed by XMM-Newton.

Results. The data analysis shows no hint for new detections at hard X-rays. The upper limits in flux herein presented constrain the energy spectrum of whatever was producing GRO J1411-64, imposing, in the framework of earlier COMPTEL observations, the existence of a peak in power output located somewhere between $300-700 \mathrm{keV}$ for the so-called low state. The Circinus Galaxy is the only source detected within the $4 \sigma$ location error of GRO J1411-64, but can be safely excluded as the possible counterpart: the extrapolation of the energy spectrum is well below the one for GRO J1411-64 at MeV energies. 22 reliable and statistically significant sources (likelihood > 10) were extracted and analyzed from XMM-Newton data. Only one of these sources, XMMU J141255.6-635932, is spectrally compatible with GRO J1411-64 although the fact the soft X-ray observations do not cover the full extent of the COMPTEL source position uncertainty make an association hard to quantify and thus risky.

Conclusions. The unique peak of the power output at high energies (hard X-rays and gamma-rays) resembles that found in the SED seen in blazars or microquasars, and might suggest that a similar scenario is at work. However, an analysis using a microquasar model consisting on a magnetized conical jet filled with relativistic electrons which radiate through synchrotron and inverse Compton scattering with star, disk, corona and synchrotron photons shows that it is hard to comply with all observational constrains. This fact and the non-detection at hard X-rays introduce an a-posteriori question mark upon the physical reality of this source, which is discussed in some detail.
\end{abstract}

Key words. X-rays: general - gamma rays: observations

\section{Introduction}

The COMPTEL experiment onboard the Compton Gamma-Ray Observatory (CGRO) surveyed the sky in the $0.75-30 \mathrm{MeV}$ energy range and detected about a dozen sources of Galactic origin. Among them, pulsars, stellar black-hole candidates, and supernova remnants were identified by the telescope (Schönfelder et al. 2000). In addition, nine unidentified sources were also reported in the first COMPTEL Catalog. Zhang et al. (2002) have reported the discovery of a new source belonging to this group. It is a variable source located near the Galactic plane. The fluxes of this COMPTEL unidentified source along the first five phases of the Compton satellite were derived by Zhang et al. (2002), and are shown, together with the corresponding confidence level of each detection, in Table 1. Time periods and effective exposures for different observations are also listed there. The best detection significance, at more than $7 \sigma$, was obtained in the $1-3 \mathrm{MeV}$ band by combining 7 viewing periods (VPs, the periods of observations in the Compton satellite) during 1995 March-July,
VP 414-424. The probability of randomly detecting a source with such high level of significance in the COMPTEL $1-3 \mathrm{MeV}$ band was estimated to be $5.4 \times 10^{-10}$; obtained by taking into account the trials for searching in all individual VPs in four energy bands from the beginning of the Compton mission in 1991 to its second reboost in 1997 (Zhang et al. 2002). The most likely source location, i.e. the maximum of the likelihood ratio distribution in the $1-3 \mathrm{MeV}$ band for the combination of VPs in 1995, is $(l, b)=\left(311.5^{\circ},-2.5^{\circ}\right)$. Therefore, the source was referred to as GRO J1411-64. The flare flux (where following the report by Zhang et al. the detection significance of $7.2 \sigma$ was measured, see the discussion below) was about $0.3 \mathrm{Crab}$, which would make of GRO J1411-64 one of the strongest sources at low galactic latitudes, second only to Crab itself. The flare duration was several months, the longest one compared to all other $\gamma$-ray sources that were monitored almost continually by COMPTEL. The rather steep spectral shape obtained while the source was flaring would predict a bright, hard X-ray source, if there is no break in the spectrum, which is explored here. 
Table 1. The GRO J1411-64 observations: effective exposures, fluxes including detection significance $\left(\chi_{1}^{2}\right)$ or $2 \sigma$ flux upper limits for the observations reported by Zhang et al.

\begin{tabular}{cccccc}
\hline \hline TJD & Effective exposure & & \multicolumn{2}{c}{ Flux $\left(10^{-5} \mathrm{ph} \mathrm{cm}^{-2} \mathrm{~s}^{-1}\right)$} \\
& days & $0.75-1 \mathrm{MeV}$ & $1-3 \mathrm{MeV}$ & $3-10 \mathrm{MeV}$ & $10-30 \mathrm{MeV}$ \\
\hline 8392-8943 (Phase 1) & 9.29 & $6.0 \pm 3.1(2.3 \sigma)$ & $<6.0$ & $1.7 \pm 1.6(1.1 \sigma)$ & $0.8 \pm 0.6(1.1 \sigma)$ \\
8943-9216 (Phase 2) & 5.87 & $4.2 \pm 3.5(1.3 \sigma)$ & $<10.1$ & $<3.9$ & $1.8 \pm 0.9(2.0 \sigma)$ \\
9216-9629 (Phase 3) & 7.45 & $<12.5$ & $6.7 \pm 3.8(1.8 \sigma)$ & $2.2 \pm 1.6(1.9 \sigma)$ & $<1.6$ \\
9629-9993 (Phase 4) & 8.42 & $13.7 \pm 3.8(4.0 \sigma)$ & $21.2 \pm 3.5(5.9 \sigma)$ & $4.6 \pm 1.6(2.9 \sigma)$ & $<1.2$ \\
9993-10371(Phase 5) & 4.23 & $18.6 \pm 4.7(4.4 \sigma)$ & $<14.7$ & $<4.8$ & $<2.6$ \\
9797-9923 (VPs 414-424) & 5.44 & $19.9 \pm 4.6(4.6 \sigma)$ & $36.6 \pm 4.7(7.7 \sigma)$ & $5.4 \pm 2.3(2.1 \sigma)$ & $<1.8(2.0 \sigma)$ \\
8392-10371(Phases 1-5) & 35.26 & $8.4 \pm 1.7(5.1 \sigma)$ & $6.1 \pm 1.6(4.0 \sigma)$ & $1.6 \pm 0.8(2.4 \sigma)$ & $0.5 \pm 0.3(2.0 \sigma)$ \\
\hline
\end{tabular}

That the source was variable within timescales of several months could be shown by two different methods by Zhang et al. (2002). On one hand, a direct statistical approach shows that if the fluxes of the individual viewing periods of the first five Phases are fitted with a constant flux, a $\chi^{2}$ value of 94.6 is derived (1-3 MeV band). That value corresponds to a significance of $4.1 \sigma$ for a variable source; alternatively, to a probability of $\sim 4.8 \times 10^{-5}$ for a constant flux. On the other hand, the variability index $I$ (Torres et al. 2001), used to normalize the measured variability of this source to the measured variability of other COMPTEL sources considered to be non-variable (so as to encompass systematics), gives a value of 5.2. This is above the $3 \sigma$-level and also classifies the source as likely variable. However, it is unclear whether this second method would apply to COMPTEL sources, due to the larger spread of indices for detected COMPTEL pulsars. In what follows, we present the results of the INTEGRAL observations of this source, as well as of XMM-Newton observation of its best location. We also comment on what can be inferred out of these observations concerning models of emission based on microquasars.

\section{Observation, data analysis, and results}

GRO J1411-64 was observed by INTEGRAL during 2004 December 30-2005 January 6. In total, 102 science windows (scws) were carried out to have $210 \mathrm{ks}$ of effective exposure. Data were taken in revolutions 270-272 using an hexagonal dithering mode. Due to the relatively small field of view (FOV) of JEMX, for most of the time the source was actually outside the scope of this instrument, and the total effective exposure in JEMX was then lowered down to roughly $83 \mathrm{ks}$. Data reduction was performed using the version 5.0 of the standard Offline Science Analysis (OSA) software, and the spectra were fitted with XSPEC of FTOOLS 5.3.1.

\subsection{Images}

No hint of signal was found for new hard X-ray sources within the location uncertainty of GRO J1411-64 from individual scws of the INTEGRAL instruments. To improve the statistics, mosaic maps were obtained for IBIS/ISGRI and JEMX by combining all data. The images of IBIS/ISGRI were produced in the energies 20-100 keV, see Fig. 1 (left panel) for the map in the $20-40 \mathrm{keV}$ band as an example. The circle shows the $4 \sigma$ of error location of GRO J1411-64 obtained by COMPTEL during its flare in 1995 (Zhang et al. 2002). This circle is centered at the location of the $\mathrm{MeV}$ source, with a radius of 2 degrees. From the possible counterparts of GRO J1411-64 discussed in Zhang et al.'s paper (2002), only the Circinus Galaxy shows up in this error region as seen by INTEGRAL. The most significant detection of
Circinus Galaxy is detected at $20-40 \mathrm{keV}$, at a confidence level of $38 \sigma$. In the mosaic map, there are 10 additional sources detected, among them the strongest one is HMXB GX301-2, with $149 \sigma$ at $20-40 \mathrm{keV}$.

The mosaic map of JEMX is shown in Fig. 1 (right panel) at $1.6-10 \mathrm{keV}$. Again the circle represents the $4 \sigma$ error contour of COMPTEL GRO J1411-64. No significant source feature is visible from within this error region. The Circinus Galaxy was most of the time outside the FOV of JEMX, and therefore it is not visible due to the relatively small exposure. Only two sources are detected from the combined JEMX data. The strongest source is $1 \mathrm{H} 1254-690$, detected with a confidence level of $122 \sigma$ in the 3-10 keV range. For SPI, only a few sources are detected at energies below $100 \mathrm{keV}$ and the maps are empty at energies above $100 \mathrm{keV}$. The Circinus Galaxy is at the $6 \sigma$ level in the $20-40 \mathrm{keV}$ range, and it is the only source detected within the location of GRO J1411-64, the region of our search.

For the sources detected in IBIS/ISGRI and JEMX, only GX 301-2, Circinus Galaxy, PSR B1509-58, 4U 1323-62, and 1H1254-690 are of interest for further investigation either due to their relatively high detection significance (like 1H1254-690 and GX 301-2) or the visible source signal extended in a relatively wide range of energies (like PSR B1509-58 and 4U 1323-62). GX 301-2 was already reported by Kreykenbohm et al. (2004), from early INTEGRAL data corresponding to 24 scws. Only one scw was at pre-periastron, with a flare detected to have its flux enhanced by a factor of 10 than the low emission state of the orbital phase. The Circinus Galaxy was also reported by Soldi (2005). For PSR B1509-58, an additional INTEGRAL observation of $1000 \mathrm{ks}$ exposure was carried out in 2005 and the data will be analyzed and reported elsewhere, we thus refrain of further comment on this source. 4U 1323-62 and 1H 1254-690 have to our knowledge not being reported earlier. Hence the preliminary analysis carried out in this paper sheds the first light on their properties in hard X-rays.

\subsection{Light curves}

The light curve for the most significant JEMX source 1H1254-690 is shown in Fig. 2 (left panel), for the IBIS/ISGRI source GX301-2 in the middle panel, and for the Circinus Galaxy in the right panel. The latter was considered a priori to be the a potential counterpart of GRO J1411-64, and was detected mainly by IBIS/ISGRI. As shown in these light curves, hard X-ray emission from the Circinus Galaxy and 1H1254-690 is rather constant, whereas that from GX 301-2 is highly variable. GX 301-2 is an eccentric hard X-ray binary system, famous for its rapid outbursts, strongly correlated to its orbital phase. The burst structure in this case is typical for GX 301-2, generally formed during the time period of orbital phase pre- and post 
Table 2. Detected INTEGRAL sources and their confidence level $(\sigma)$, flux and flux error (in units of ct/s), in the different energy ranges explored (in $\mathrm{keV}$ ).

\begin{tabular}{llllllllll}
\hline \hline \multicolumn{1}{c}{ Source name } & \multicolumn{4}{c}{$20-40 \mathrm{keV}$} & \multicolumn{3}{c}{$40-80 \mathrm{keV}$} & \multicolumn{3}{c}{$80-120 \mathrm{keV}$} \\
& $\sigma$ & Flux & Flux error & $\sigma$ & Flux & Flux error & $\sigma$ & Flux & Flux error \\
\hline GX 301-2 & 149 & 10.72 & 0.07 & 12 & 0.82 & 0.07 & $\ldots$ & $\ldots$ & $\ldots$ \\
Circinus Galaxy & 38 & 1.35 & 0.03 & 20 & 0.75 & 0.04 & $\ldots$ & $\ldots$ & $\ldots$ \\
4U 1626-67 & 20 & 1.95 & 0.10 & $\ldots$ & $\ldots$ & $\ldots$ & $\ldots$ & $\ldots$ & $\ldots$ \\
PSR B1509-58 & 16 & 0.82 & 0.05 & 13 & 0.66 & 0.05 & 8 & 0.28 & 0.04 \\
H 1538-522 & 12 & 2.06 & 0.16 & $\ldots$ & $\ldots$ & $\ldots$ & $\ldots$ & $\ldots$ & $\ldots$ \\
1E 1145.1-6141 & 10 & 1.77 & 0.18 & $\ldots$ & $\ldots$ & $\ldots$ & $\ldots$ & $\ldots$ & $\ldots$ \\
Cir X-1 & 9 & 0.58 & 0.06 & $\ldots$ & $\ldots$ & $\ldots$ & $\ldots$ & $\ldots$ & $\ldots$ \\
NGC 4945 & 7 & 1.31 & 0.19 & $\ldots$ & $\ldots$ & $\ldots$ & $\ldots$ & $\ldots$ & $\ldots$ \\
2RXP J130159.5-63580 & 7 & 0.32 & 0.05 & $\ldots$ & $\ldots$ & $\ldots$ & $\ldots$ & $\ldots$ & $\ldots$ \\
4U 1323-62 & 34 & 1.36 & 0.04 & 17 & 0.73 & 0.04 & $\ldots$ & $\ldots$ & $\ldots$ \\
\hline
\end{tabular}
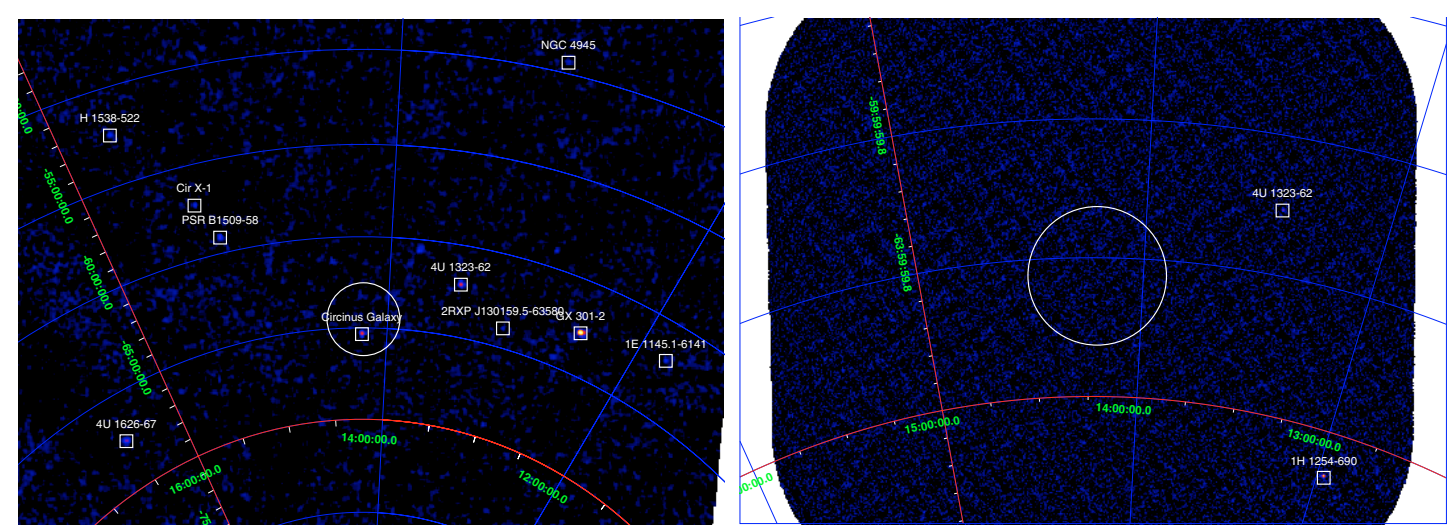

Fig. 1. Left: sky map of the GRO J1411-64 region as seen by IBIS/ISGRI in the 20-40 keV range, by combining all data obtained in the observations performed during 2004 December 30 to 2005 January 6. See Table 2 for details of each detected source. Right: sky map of the GRO J1411-64 region as seen by JEMX in 1.6-10 keV range, by combining all data obtained in the observations performed during 2004 December 30 to 2005 January 6.

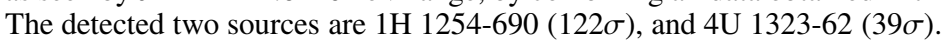
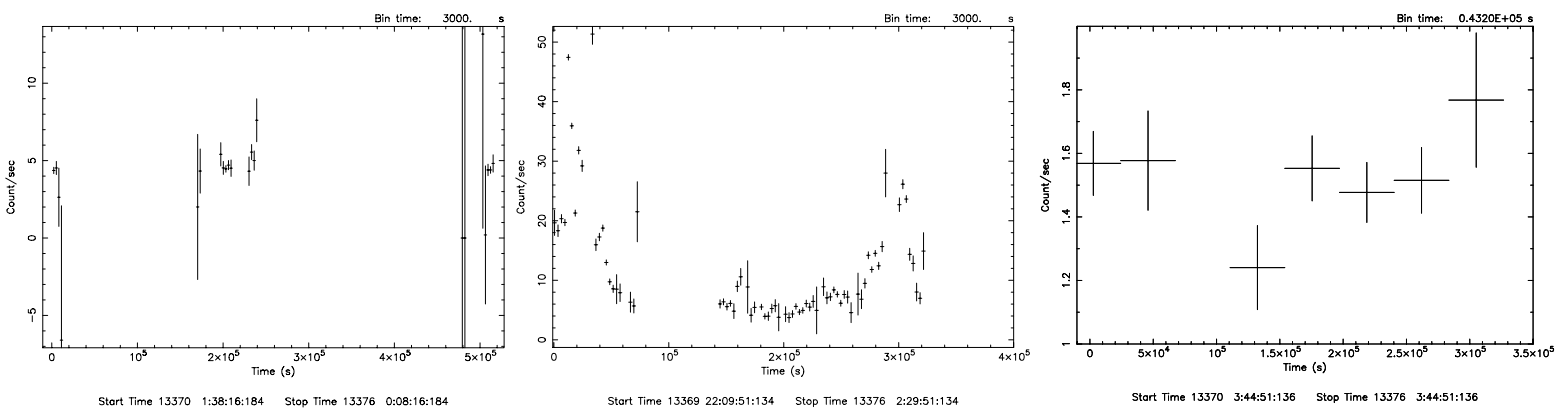

Fig. 2. Left: light curve of $1 \mathrm{H} 1254-690$ with each bin averaged over $3000 \mathrm{~s}$, observed by JEMX in the 1.6-10 keV range. Middle: light curve of GX 310-2 with each bin averaged over $3000 \mathrm{~s}$, observed by IBIS/ISGRI in the 20-40 keV range. Right: light curve of the Circinus Galaxy with each bin averaged over half day, observed by IBIS/ISGRI in the 20-40 keV range.

periastron (Koh et al. 1997; Kreykenbohm et al. 2004; La Barbera et al. 2005). This source is, nonetheless, far from the region of GRO J1411-64, and thus it may not be related to it.

\subsection{Energy spectra}

Since the sources are mainly detected by the individual instruments of INTEGRAL, the energy spectra of 1H1254-690, GX301-2 and the Circinus Galaxy, were analyzed with the observational data from the corresponding instruments that detected them. The data were summed up at the well calibrated energies of 3-20 keV for JEMX, and photons with energies $\geq 20 \mathrm{keV}$ detected by IBIS/ISGRI were used in spectral fitting.
1H1254-690 is well fitted by the cutoffpl model (see Eq. (1)) in XSPEC,

$f(E)=K E^{-\alpha} \exp \left(-E / E_{\mathrm{c}}\right)$

where $K$ is a normalization factor $\left(\mathrm{keV}^{-1} \mathrm{~cm}^{-2} \mathrm{~s}^{-1}\right)$ at $1 \mathrm{keV}, \alpha$ is the power law photon index and $E_{\mathrm{c}}$ is the energy of the exponential rolloff $(\mathrm{keV})$. This model produces a reduced $\chi^{2}$ of 1.2 for 133 d.o.f. (see Fig. 3). The cutoffpl model represents well the spectral data for GX 301-2 as well (see Fig. 3, middle panel), with a reduced $\chi^{2}$ of 0.9 (17 d.o.f.). The former reported cyclotron resonance scattering feature (CRSF) at either $45 \mathrm{keV}$ or $35 \mathrm{keV}$ (La Barbera et al. 2005; Kreykenbohm et al. 2004) are not visible in ISGRI, most probably due to the relatively poor statistics of the data. The Circinus Galaxy was investigated by 

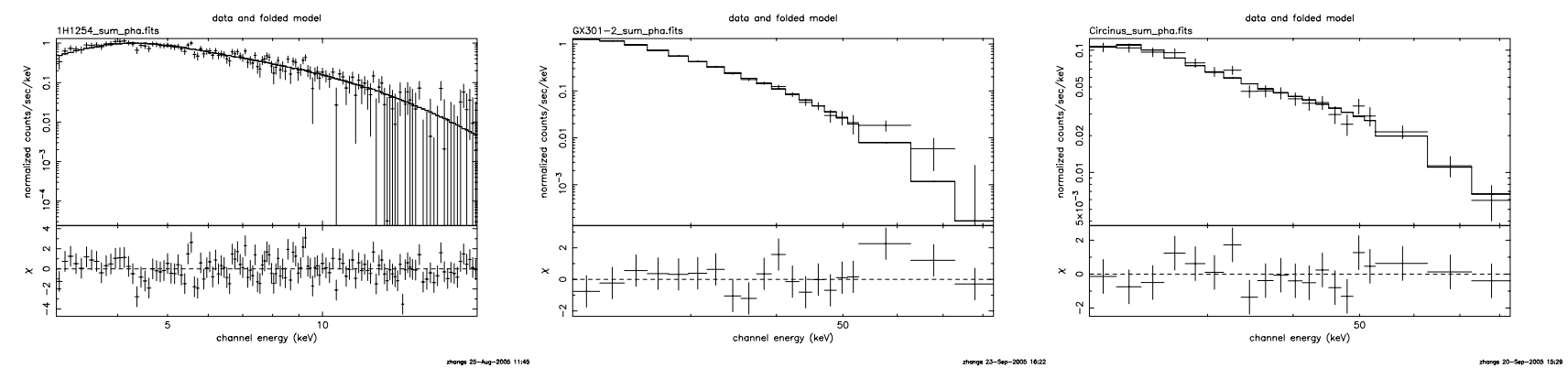

Fig. 3. Left: spectrum of 1H1254-690 from the JEMX combined data. The fit model is cutoffpl in XSPEC. Middle: spectrum of GX 301-2 from IBSI/ISGRI combined data. The fit model is cutoffpl in XSPEC. Right: spectrum of the Circinus Galaxy from IBIS/ISGRI combined data. The fit model is cutoffpl plus wabs in XSPEC.

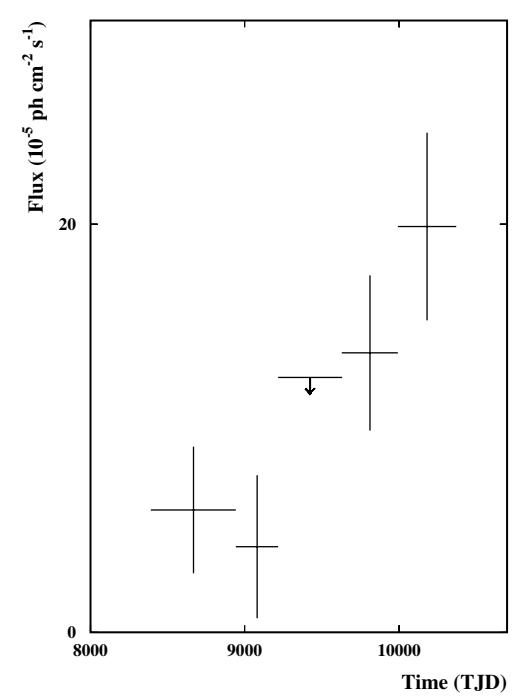

Fig. 4. Light curve of GRO J1411-64 as observed by COMPTEL at $0.75-1 \mathrm{MeV}$ band. The error bar is $1 \sigma$ and upper limit $2 \sigma$. These data points include the 7 viewing periods when the source was flaring.

Soldi et al. (2005); models of cutoffpl plus wabs in XSPEC can fit the data well, with a reduced $\chi^{2}$ of 1.1 (7 d.o.f.). The resulting parameters from Soldi et al. were referred to in our fitting of the IBIS/ISGRI observational data as follows: $N_{\mathrm{H}}$ and $E_{\mathrm{c}}$ were fixed to the Soldi et al.'s values of $400 \times 10^{22}$ atoms $\mathrm{cm}^{-2}$ and $50 \mathrm{keV}$, respectively. The fit results in a reduced $\chi^{2}$ of 0.72 (18 d.o.f., see Fig. 3, right panel). The spectral index is then derived as $1.82 \pm$ 0.09 , consistent with Soldi et al.'s value of $1.8_{-0.5}^{+0.4}$.

\subsection{Hard X-rays to MeV spectral constraints}

The light curve of GRO J1411-64 is shown in Fig. 4, including the flare period, showing that if there is emission out of the flare it is likely steady in $0.75-1 \mathrm{MeV}$ band, and requires, in absence of a peak in the spectral energy distribution, a relatively bright INTEGRAL source. During its low state, the source was detected at $\sim 4 \sigma$ by COMPTEL in the energies of $0.75-1 \mathrm{MeV}$. In Fig. 5, a map of GRO J1411-64 is presented based on data only from the low state in this energy band. The corresponding spectrum of the low state can be represented by a power law shape with spectral index $2.5_{-0.4}^{+0.6}$ (see Fig. 6).

The ISGRI/SPI upper limits combined to spectra of both flare/low states shows the existence of a maximum in the power output at hard X-rays. If the source was at its flare state, the power maximum in the combined spectrum is around

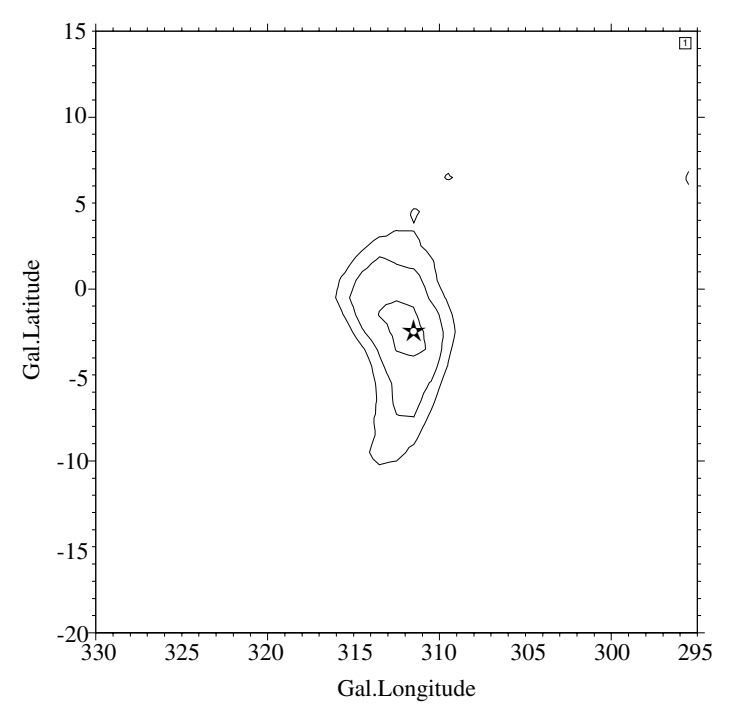

Fig. 5. The skymap of GRO J1411-64 as observed by COMPTEL in $0.75-1 \mathrm{MeV}$ during 1991-1996, not including the flare period of 4 months in 1995. The star represents the best-guessed source location. The contour lines start at a detection significance level of $3 \sigma$ with steps of $0.5 \sigma$.

500-700 keV, otherwise, if the source was at the low state as defined by Zhang et al. (2002), the maximum is somewhere between 300-700 keV.

In addition, a combination of the best-fit spectrum of the Circinus Galaxy at hard X-rays with the one detected at MeV energies of GRO J1411-64, suggests that this Seyfert galaxy is not the counterpart of the MeV source: its spectral extrapolation is well below the ones at MeV energies for both states. Such a unique power maximum at high energies, might remind the second peak in the SED of a blazar or microblazar, which represent the emission component originating in the inverse Compton process of the soft photon off the highly relativistic leptons within the jet. We explore briefly this possibility in the ending discussion.

\section{XMM-Newton observations of the innermost region of the COMPTEL source}

The COMPTEL uncertainty in the position of the source, and the fact that no clear counterpart has been found in the INTEGRAL range, makes a direct search at $\mathrm{keV}$ energies more difficult. In any case, we have conducted a search of the innermost region of the COMPTEL location, in order to avoid missing any obvious faint counterpart that may be residing there, and that might have 
Table 3. Results for the spectral fitting of GX 301-3, the Circinux Galaxy, and 1H 1254-690. The values without error bars show the corresponding parameters that can not be constrained from the data.

\begin{tabular}{lllllll}
\hline \multicolumn{1}{c}{ Source name } & \multicolumn{1}{c}{$\begin{array}{c}N_{\mathrm{H}} \\
\text { atoms cm }\end{array}$} & \multicolumn{1}{c}{$\alpha$} & $\begin{array}{c}E_{\mathrm{c}} \\
\mathrm{keV}\end{array}$ & $\begin{array}{c}K \\
\times \mathrm{keV}^{-1} \mathrm{~cm}^{-2} \mathrm{~s}^{-1}\end{array}$ & reduced $\chi^{2}$ & d.o.f. \\
\hline GX 301-2 & $\ldots$ & $2.1_{-0.6}^{+0.2}$ & $4.61_{-0.02}^{+0.15}$ & $4 \times 10^{-4}$ & 0.9 & 17 \\
Circinus Galaxy & 400 (fixed) & $1.82 \pm 0.09$ & 50 (fixed) & $0.11_{0.03}^{+0.04}$ & 0.7 & 18 \\
$1 \mathrm{H} \mathrm{1254-690}$ & $\ldots$ & $1.64 \pm 0.33$ & $4.5_{-0.9}^{+1.3}$ & $0.62 \pm 0.20$ & 1.2 & 133 \\
\hline
\end{tabular}

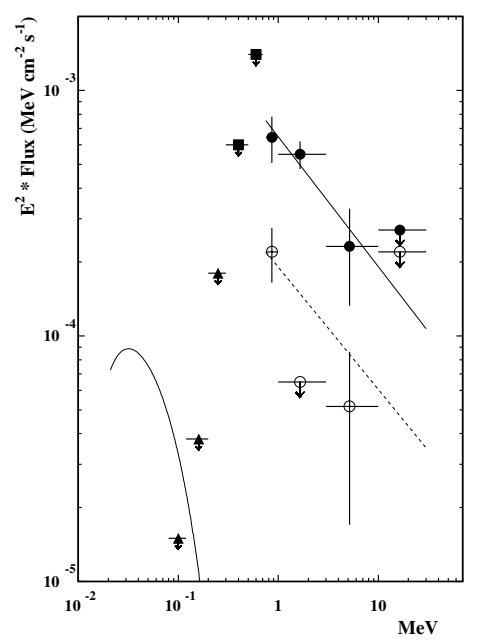

Fig. 6. Combined energy spectrum of GRO J1411-64. Filled (open) circles represent flare (low) states of GRO J1411-64 as observed by COMPTEL at MeV energies. The corresponding power law shapes (solid line for the flare state, dashed line for the low state), are compared to $2 \sigma$ upper limits obtained from IBIS/ISGRI (triangles) and SPI (squares). The solid curve at low energies is the energy spectrum of Circinus Galaxy derived from the IBIS/ISGRI data. The error bar is $1 \sigma$ and upper limit $2 \sigma$.

been missed by the INTEGRAL observations. The best position of the COMPTEL source (centered at $(l, b)=311.5^{\circ},-2.5^{\circ}$ ) had not been observed by any recent X-ray mission (Chandra, ASCA, XTE, or XMM-Newton) until now. HEASARC archival search showed no matches for past X-ray missions (Einstein, Ginga, Copernicus, etc.) either. In addition, there is no ROSAT pointed observation. ROSAT only reported three faint sources about 13 to 20 arcmin from the center of the COMPTEL source, in the ROSAT Faint Sources Catalog (rassfsc), with only $\sim 400 \mathrm{~s}$ exposure.

The best localization of COMPTEL source GRO 141164 position was observed with XMM-Newton during revolution 960 on the 7th of March of 2005 (Observation ID: 0204010101). The EPIC instrument cameras, MOS1, MOS 2 and pn, were operated in full window (imaging) mode, with thick filters to prevent optical contamination from bright stars in the field. The exposure times for each camera were 18, 18 and $22 \mathrm{ks}$ for MOS1, MOS2 and pn respectively. The data were pipelineprocessed with the XMM-Newton Science Analysis Software (SAS) version 6.1. After removal of background flares, a total of 15.8, 15.8 and $14.6 \mathrm{ks}$ of good data survived for MOS1, MOS2 and pn respectively.

A total of 31 X-ray sources were formally detected by the SAS source detection algorithm in the EPIC data. Nine of these were excluded due to detector defects and other artifacts, in a careful inspection. The resulting 22 reliable and statistically significant sources (likelihood $>10$ ) are shown in Fig. 7, and their main properties catalogued in Table 4. Due to the low

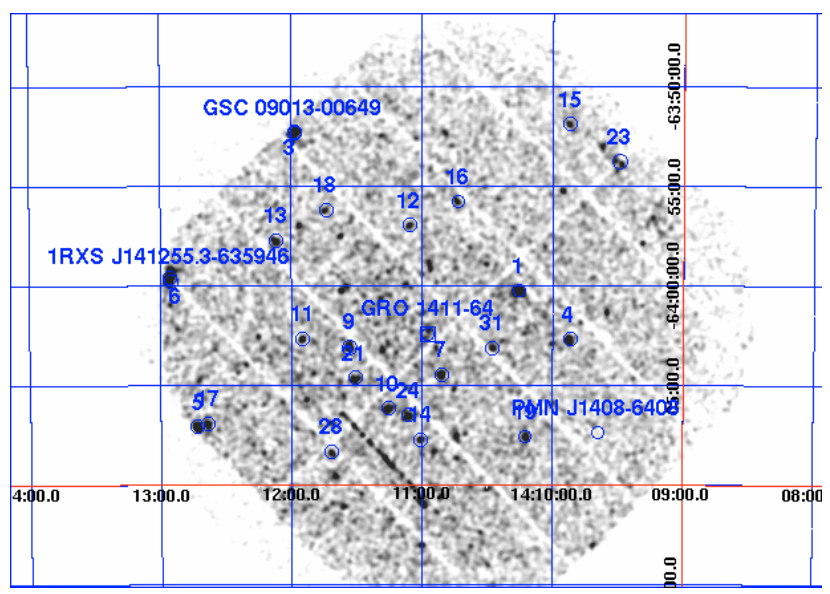

Fig. 7. XMM-Newton EPIC image (combining all 3 cameras), where detected sources and previously catalogued sources have been labeled. The centroid of the COMPTEL source GRO 1411-64 is marked with a square box, the error contour being larger than the image itself.

Galactic latitude of this observation, no attempt was made to refine the astrometry provided by the spacecraft attitude solution against optical sources. This is known to lead to systematic uncertainties in the position of the sources of up to a few arcsec at most.

X-ray spectra of all these sources were extracted from the 3 EPIC cameras, using circular regions with radii that optimize the signal to noise in every detector. Background spectra were extracted in nearby circular source-free regions that avoid CCD gaps. Calibration files (redistribution RMF and effective area ARF) were generated for every spectrum, using the SAS tasks rmfgen and arfgen.

In Fig. 8 we display the X-ray hardness ratio $\left(H R_{2}=(H-\right.$ $S) /(H+S)$, where $H$ and $S$ are the flux in the $2.0-4.5 \mathrm{keV}$ and 0.5-2.0 keV bands respectively) versus the total EPIC-pn source counts of the sources detected in the EPIC-pn camera. Given the large Galactic column along this direction $\left(N_{\mathrm{H}} \sim 1.13 \times\right.$ $\left.10^{22} \mathrm{~cm}^{-2}\right)$, all extragalactic sources are expected to have a high value of the hardness ratio $H R_{2}>0$. Extragalactic sources are therefore a sizeable fraction of the sources detected.

We now concentrate on a careful analysis of the spectra of the 4 likely Galactic sources (small hardness ratio) with more than 50 EPIC-pn source counts.

Source XMMU J141015.5-640015 (label 1) is well fitted by a simple black body spectrum, with no absorption. The best fit for the temperature is $k T \sim 270_{-30}^{+40} \mathrm{eV}$, which points towards a nearby active stellar corona.

Source XMMU J141157.9-635216 (label 3) matches the position of the star GSC 09013-00649. The X-ray spectrum is well fit by a black body at $k T \sim 250_{-70}^{+40} \mathrm{eV}$, modified by a small amount of photoelectric absorption 
Table 4. Catalogue of the sources detected in the XMM-Newton EPIC-pn camera.

\begin{tabular}{|c|c|c|c|c|c|c|}
\hline \multicolumn{2}{|l|}{ Source } & \multicolumn{3}{|c|}{ Position } & EPIC-pn $0.2-12 \mathrm{keV}$ & EPIC-pn $\mathrm{HR}_{2}$ \\
\hline Name & Label & RA(J2000) & $\operatorname{Dec}(\mathrm{J} 2000)$ & Error (arcsec) & Source counts & \\
\hline XMMU J141015.5-640015 & 1 & $14: 10: 15.5$ & $-64: 00: 15.7$ & 0.4 & $67 \pm 9$ & $-0.84 \pm 0.12$ \\
\hline XMMU J141157.9-635216 & 3 & $14: 11: 57.9$ & $-63: 52: 16.8$ & 0.4 & $110 \pm 15$ & $-0.80 \pm 0.10$ \\
\hline XMMU J140952.3-640243 & 4 & $14: 09: 52.3$ & $-64: 02: 43.2$ & 0.7 & $14 \pm 5$ & $-0.96 \pm 0.21$ \\
\hline XMMU J141243.2-640701 & 5 & $14: 12: 43.2$ & $-64: 07: 01.4$ & 0.6 & $74 \pm 10$ & $-0.21 \pm 0.17$ \\
\hline XMMU J141255.6-635929 & 6 & $14: 12: 55.6$ & $-63: 59: 29.8$ & 0.3 & $173 \pm 15$ & $-0.89 \pm 0.05$ \\
\hline XMMU J141051.2-640429 & 7 & $14: 10: 51.2$ & $-64: 04: 29.2$ & 0.7 & $33 \pm 7$ & $-1.00 \pm 0.03$ \\
\hline XMMU J141133.3-640304 & 9 & $14: 11: 33.3$ & $-64: 03: 04.3$ & 0.9 & $23 \pm 6$ & $-0.28 \pm 0.35$ \\
\hline XMMU J141115.6-640610 & 10 & $14: 11: 15.6$ & $-64: 06: 10.3$ & 1.2 & $31 \pm 7$ & $-0.92 \pm 0.15$ \\
\hline XMMU J141154.8-640238 & 11 & $14: 11: 54.8$ & $-64: 02: 38.5$ & 1.0 & $28 \pm 7$ & $-0.95 \pm 0.17$ \\
\hline XMMU J141105.6-635659 & 12 & $14: 11: 05.6$ & $-63: 56: 59.2$ & 1.7 & $19 \pm 6$ & $-0.92 \pm 0.20$ \\
\hline XMMU J141206.6-635743 & 13 & 14:12:06.6 & $-63: 57: 43.2$ & 1.0 & $19 \pm 6$ & $-1.00 \pm 0.13$ \\
\hline XMMU J141100.7-640744 & 14 & 14:11:00.7 & $-64: 07: 44.7$ & 1.5 & $25 \pm 6$ & $-0.86 \pm 0.23$ \\
\hline XMMU J140951.9-635156 & 15 & 14:09:51.9 & $-63: 51: 56.9$ & 1.3 & $20 \pm 6$ & $+0.55 \pm 0.50$ \\
\hline XMMU J141043.3-635547 & 16 & $14: 10: 43.3$ & $-63: 55: 47.6$ & 1.4 & $13 \pm 5$ & $+0.81 \pm 0.50$ \\
\hline XMMU J141238.5-640652 & 17 & $14: 12: 38.5$ & $-64: 06: 52.0$ & 1.2 & $27 \pm 7$ & $+0.11 \pm 0.30$ \\
\hline XMMU J141143.8-635610 & 18 & $14: 11: 43.8$ & $-63: 56: 10.4$ & 1.2 & $13 \pm 5$ & $-0.25 \pm 0.43$ \\
\hline XMMU J141012.6-640736 & 19 & $14: 10: 12.6$ & $-64: 07: 36.6$ & 1.5 & $15 \pm 5$ & $-0.04 \pm 0.36$ \\
\hline XMMU J141130.8-640439 & 21 & $14: 11: 30.8$ & $-64: 04: 39.8$ & 1.6 & $18 \pm 5$ & $-0.60 \pm 0.55$ \\
\hline XMMU J141106.3-640632 & 24 & 14:11:06.3 & $-64: 06: 32.1$ & 1.1 & $11 \pm 5$ & $-0.84 \pm 0.30$ \\
\hline
\end{tabular}

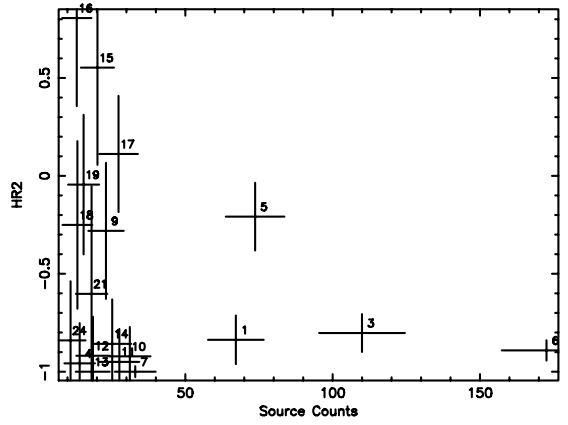

Fig. 8. EPIC pn hardness ratio $H R_{2}$ (see text for details) versus source counts in that detector.

$\left(N_{\mathrm{H}} \sim 0.15_{-0.14}^{+0.3} \times 10^{22} \mathrm{~cm}^{-2}\right)$, and is therefore consistent with a Galactic star with some intervening absorption.

Source XMMU J141243.2-640701 (label 5), the hardest of the spectra analyzed, is most likely an extragalactic AGN, as the $\mathrm{X}$-ray spectrum is appropriately fit by a power law with energy spectral index $\Gamma \sim 1.6_{-1.1}^{+0.8}$ and absorbing column $N_{\mathrm{H}}=0.7_{-0.7}^{+0.6} \times$ $10^{22} \mathrm{~cm}^{-2}$, consistent with the Galactic column in that direction.

Source XMMU J141255.6-635929 (label 6, coincident with ROS AT source 1RXS J141255.3-635946 within 16 arcsec) is very intriguing. In the discussion that follows, we consider only the energy range from $0.2-7 \mathrm{keV}$, for which we have $51 \mathrm{spec}-$ tral bins with 10 counts each. The source has a small hardness ratio, indicating a Galactic origin. However, its spectrum is not well fit by an absorbed thermal model $\left(\chi^{2}=75.8\right.$, for 48 degrees of freedom) which leaves large and correlated residuals both at low and high energies. The low energy residuals can be removed if we restrict the fit to below $2 \mathrm{keV}$, but then a significant high energy tail is apparent. The origin of this high energy tail can be addressed in at least two ways. First, we can fit a second black body spectrum with a different absorption. This gives a formally acceptable fit to the whole spectrum $\left(\chi^{2}=49.70\right.$ for 46 degrees of freedom), with a low-temperature black body with $k T=121_{-39}^{+30} \mathrm{eV}$ absorbed by $N_{\mathrm{H}}=0.39_{-0.17}^{+0.29} \times 10^{22} \mathrm{~cm}^{-2}$ and a second much hotter black body with $k T=432_{-120}^{+404} \mathrm{eV}$ absorbed by a highly uncertain column $N_{\mathrm{H}}=2_{-2}^{+6} \times 10^{22} \mathrm{~cm}^{-2}$

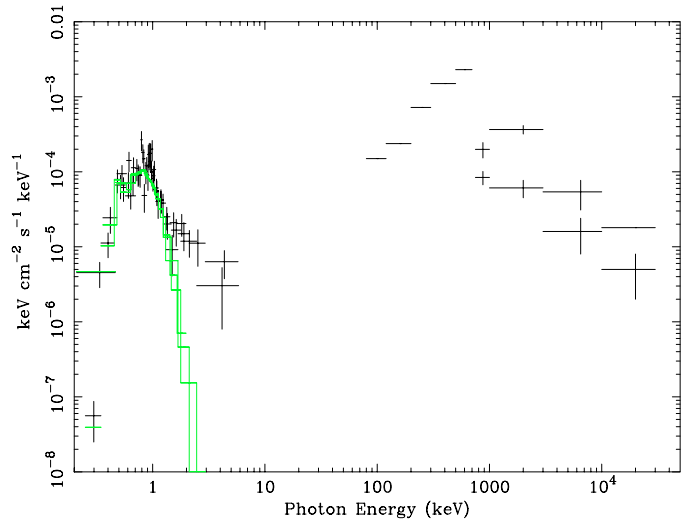

Fig. 9. XMM-Newton unfolded spectrum of the X-ray source XMMU J141255.6-635932. The model shown is only the thermal component in the X-ray spectrum. The COMPTEL detections and the INTEGRAL upper limits are also shown at high energies, with horizontal bars denoting $2 \sigma$ upper limits.

(as customary, errors denote $90 \%$ uncertainty for one single interesting parameter). This second black body appears very large for a second active star, positionally confused with the former. Although we cannot reject this option, we find it very unlikely. An additional possibility is that the hard excess in this source is modeled as a power law. The fit to a black body plus a power law, both absorbed by intervening material, is also very good with $\chi^{2}=51.90$ for 46 degrees of freedom. In this case $k T=106_{-30}^{+23}$, $N_{\mathrm{H}}=0.48_{-0.18}^{+0.42} \times 10^{22} \mathrm{~cm}^{-2}$ and a highly uncertain photon index of $\Gamma=2.3_{-0.8}^{+1.2}$. Attempts to fit the soft part of the spectrum with a steep power law resulted in extremely large values of the spectral slope $(\sim 7-8)$ and significant residuals accompanied by a large increase in the $\chi^{2}$.

In Fig. 9 we plot the unfolded spectrum (largely independent of the model fitted) for XMMU J141255.6-635932, along with the best fit model (with these parameters), the COMPTEL detections and the INTEGRAL upper limits from Table 1. The hard excess exhibited by the XMM-Newton data is apparent in that figure, and might be suggestive of a large Compton bump that would peak in the several $\sim 100 \mathrm{keV}$ region, fitting well with the 


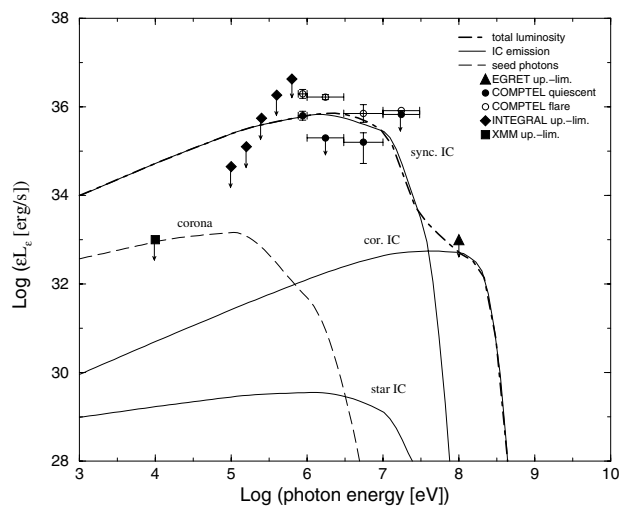

Fig. 10. A microquasar model in the light of observational constraints. See text for details.

COMPTEL detections. However, the fact that the XMM-Newton image does not cover the full COMPTEL source location and the non-detection by INTEGRAL of any reliable counterpart, would make the assumption that XMMU J141255.6-635932 is the counterpart to GRO 1411-64, although spectrally consistent, only tentative and risky.

\section{Discussion and concluding remarks}

We have applied to the non-simultaneous data from XMM-Newton (treating them as an upper limit ${ }^{1}$ ), INTEGRAL and COMPTEL the microquasar model presented by Bosch-Ramon et al. (2006). This model consists of a magnetized conical jet filled with relativistic electrons which radiate through synchrotron and inverse Compton scattering with star, disk, corona and synchrotron photons. These electrons lose energy mainly through adiabatical losses, as a first order approximation. At the top of Table 5, parameter values for a typical microquasar system and jet geometry are given (Bosch-Ramon et al. 2006). We have considered different values within the range open for the free parameters finding that it is not possible to obtain a simple microquasar model that could fit the SED. In particular, in Fig. 10 we show a test case with the free parameters fixed to the values presented in Table 5, at the bottom. We note that, since the computed SED in Fig. 10 is dominated in the gamma-ray band by SSC emission, the model results would also apply for a low mass microquasar.

If the COMPTEL source is to be among the group of low luminosity soft X-ray sources and be detectable at energies about $1 \mathrm{MeV}$, a very hard photon index of about 0.67 (i.e. $n_{v} \propto$ $v^{-0.67}$ ) will be required in the energy range $10-1000 \mathrm{keV}$ assuming a power-law energy distribution for the radiation. Even for a strongly absorbed soft X-ray counterpart (see, e.g. Walter et al. 2003), or a soft X-ray counterpart out of the XMM-Newton field observed, the INTEGRAL upper-limits impose also a very hard photon index of about 1 in the range $100-1000 \mathrm{keV}$, whereas radiation at higher energies is strongly constrained by EGRET observations. In the context of microquasars, if the particle spectrum follows a power-law, it is difficult to explain such narrow band emission concentrated in the $\mathrm{MeV}$ range.

1 This is actually a not well-justified a priori assumption, since the coverage of XMM-Newton observations is much smaller than that of INTEGRAL, and it is imposed by fiat, in the knowledge that the constraints imposed below do not crucially depend on the XMM-Newton point, but rather on the steepness of the INTEGRAL upper limits.
Table 5. Parameter values for GRO 1411-64.

\begin{tabular}{cl}
\hline \hline Parameter & Values \\
\hline Stellar bolometric luminosity [erg s & -1 \\
Distance from the apex of the jet to the compact object $[\mathrm{cm}]$ & $5 \times 10^{7}$ \\
Initial jet radius [cm] & $5 \times 10^{6}$ \\
Orbital radius [cm] & $3 \times 10^{12}$ \\
Viewing angle to the axis of the jet [ $\left.{ }^{\circ}\right]$ & 45 \\
Jet Lorentz factor & 1.2 \\
\hline Jet leptonic kinetic luminosity [erg s ${ }^{-1}$ ] & $3 \times 10^{35}$ \\
Maximum electron Lorentz factor (jet frame) & $5 \times 10^{2}$ \\
Maximum magnetic field [G] & 8000 \\
Electron power-law index & 1.5 \\
Total corona luminosity [erg s ${ }^{-1}$ ] & $3 \times 10^{33}$ \\
\hline
\end{tabular}

Concerning other transients spatially coincident with the INTEGRAL observations we mention 2S 1417-624, a Be/X-ray binary pulsar system, with a Be star of $>5.9 M_{\odot}$ for a neutron star of $1.4 M_{\odot}$. BATSE observed a huge outburst of this source at hard X-rays (pulsations up to $100 \mathrm{keV}$ were detected), which started on August 26, 1994 and lasted 110 days (Finger et al. 1996). COMPTEL observed the source twice (VPs 402, 402.5) during this period but did not detect it. During the next 200 days, 2S 1417-624 showed 5 smaller outbursts (Finger et al. 1996), which coincide in time with the significant COMPTEL detection of the MeV source in 1995. During the huge outburst in hard X-rays, the pulsed luminosity was found to be much less than it would be estimated from the spin-up rate. This implies that most of the power output is either unpulsed or outside of the hard X-ray range (Finger et al. 1996). Assuming 2S 1417-624 as counterpart of the COMPTEL source, its $0.75-10 \mathrm{MeV}$ luminosity would be $2.5 \times 10^{37} \mathrm{erg} \mathrm{s}^{-1}$, obtained by using its upperlimit distance of $11.1 \mathrm{kpc}$. This luminosity is comparable to the pulsed one of $2.2 \times 10^{37} \mathrm{erg} \mathrm{s}^{-1}$. $2 \mathrm{~S} 1417-624$ as counterpart might show an anticorrelation of the $\mathrm{X}$ - and $\gamma$-ray emission as proposed for other sources (Romero et al. 2001). GS 1354-645, also in the field, is a transient black-hole X-ray binary (XRB) system. However, no X-ray observations of GS 1354-624 during the activity period reported for the COMPTEL source are reported. Since INTEGRAL did not detect these sources during our campaign, we cannot open judgment about the possibility for an outbursting system such as these to be the counterpart of the reported detection.

Failing to associate a plausible counterpart of GRO J1411-64 at the hard X-rays from our INTEGRAL observations, we have looked again at the initially reported gamma-ray properties of GRO J1411-64. In Table 1, we have already expanded beyond the initially reported source characteristics from Zhang et al. (2002) by including the individual detection significances. If we apply a rather conservative detection criterion (significance threshold) of at least $4 \sigma$ in an individual measurement, a firm detection can be claimed only in the so-called flare state, which was attributed to occur within CGRO viewing periods 414-424. During this flare period as well as any other investigated time span, a detection threshold of at least $4 \sigma$ in the individual measurement allows the determination of the photon spectrum from significantly measured data points, i.e., in two energy bands only, from $0.75-1 \mathrm{MeV}$ and $1-3 \mathrm{MeV}$, respectively. An accordingly determined spectrum yields an index of $2.18 \pm 0.2$, substantially harder than the previous reported index of $2.53 \pm 0.2$ taking into account lower confidence data points. If we fit the range from 0.75 to $10 \mathrm{MeV}$ with a statistically weighted power law fit, an index of $2.48 \pm 0.2$ will be determined. Therefore, the 


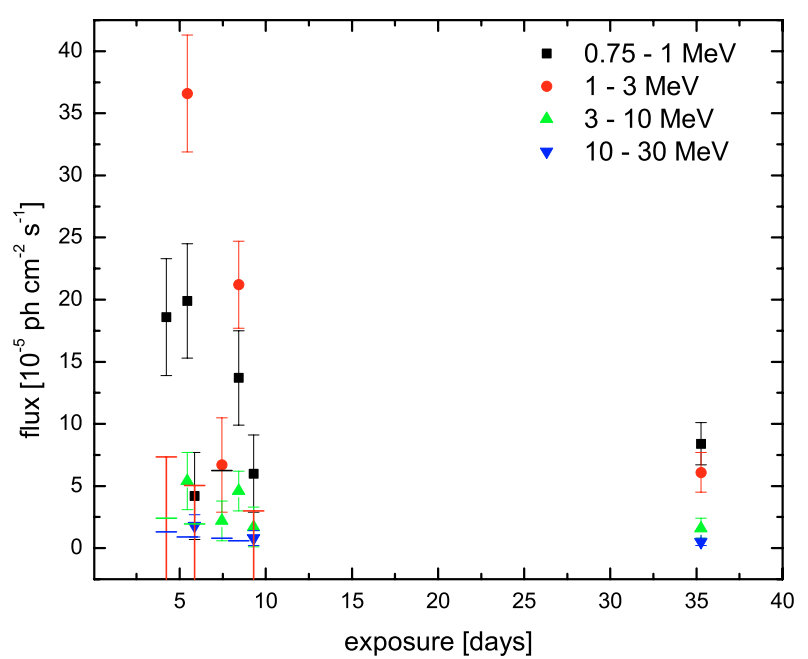

Fig. 11. The original observations of GRO J1411-64 as reported in Zhang et al. (2002), see Table 1. The dependence from the individual measurements from the underlaying exposure is easily seen, as is the different character in the phase 4 vs. phase 5 measurements, which exhibit the largest flux values, but missing a $1-3 \mathrm{MeV}$ flux in case of the phase 5 measurement.

dependence from the individual measurements becomes crucial for determining a spectral slope.

If we compare the observations of GRO J1411-64, as made in Fig. 11, indicating the measured flux/upper limit in the four energy bands as a function from the respective exposure, other characteristics can be seen. Here, we convert the $2 \sigma$ upper limits from Zhang et al. (2002) into $1 \sigma$ upper limits, in order to prevent quoting upper limits based on higher statistical confidence than certain flux values from Table 1 . One clearly sees that the flaring phenomenon related to the CGRO observation periods 414-424, is not based on any extraordinary long or short exposure when compared with other observations. It can also be seen that the best signals originated from the two lower energetic band in COMPTEL, as already indicated from the individual detection significances. Finally, whereas there is a clear indication of the spectral behavior being a steep spectrum source in the phase 4 observations, there is a remarkable difference in the phase 5 observation: Here, the largest flux has been quoted from the 0.75 to $1 \mathrm{MeV}$ band. However, in the immediate neighboring energy band (1-3 MeV), only an upper limit has been obtained. Comparing for the so-called flare phase, where the flux in the $0.75-1 \mathrm{MeV}$ band is clearly exceeded by the $1-3 \mathrm{MeV}$ band data, we should perhaps reconsider the flare phenomenon: on one hand, the actual low energy band data point in phase 5 is measured at higher flux and better statistical significance, on the other hand the 1-3 MeV flux measurement is missing, indicating that the spectrum was even more extreme in the phase 5, and clearly different from phase 4 , and any other considered time span quoted in Zhang et al. (2002). This is also seen in the total superposition, where the four energy bands line up in an more usual behavior, i.e., any subsequent higher energetic band exhibit a lower flux. In the light of such reassessment we note that a flare phenomenon is not consistently to be described as either spectral hardening or softening compared to the sparse overall data from the region. We note, however, that the indication for this phenomenon is primarily based on data with the lowest effective exposure. Thus, the distinction of different state becomes blurry.

GLAST observations would help improving the location of the $\mathrm{MeV}$ source if radiation at higher energies is not completely suppressed, and would open the door for more efficient multiwavelength searches of the counterpart. However, it is true that the nature of this COMPTEL source might not be constrained further if this detection was a one-time only transient phenomena. GLAST will only be able to help if a candidate counterpart is caught in the act (flaring/quiescent state of an AGN or a more rare galactic object). Having at hand GLAST observations, in any case, will make our currently reported investigation to naturally fit into the testing of any hypothesis on the nature of GRO J1614-64.

Acknowledgements. We thank Dr. M. T. Ceballos for her help with the XMM-Newton data. DFT was partially supported by NASA and acknowledges the Lawrence Livemore National Laboratory, where the initial part of this research was done. S. Zhang was subsidized by the Special Funds for Major State Basic Research Projects and by the National Natural Science Foundation of China. X.B. and A.C. were financially supported for this research by the Ministerio de Educación y Ciencia (Spain), under project ESP2003-00812. V.B.-R. and J.M.P. have been supported by Ministerio de Educación y Ciencia (Spain) under grant AYA-2004-07171-C02-01, as well as additional support from the European Regional Development Fund (ERDF/FEDER). V.B.-R. has been additionally supported by the DGI of the Ministerio de (Spain) under the fellowship BES-2002-2699. GER was supported by grants PIP $5375 \mathrm{y}$ PICT 03-13291.

\section{References}

Bosch-Ramon, V., Paredes, J. M., Romero, G. E., \& Torres, D. F. 2006, A\&A, 446, 1081

La Barbera, A., Segreto, A., Santangelo, A., et al. 2005, A\&A, 438, 617 Finger, M. H., Wilson, R. B., \& Chakrabarty, D. 1996, A\&AS, 120, 209 Koh, D. T., Bildsten, L., Chakrabarty, D., et al. 1997, ApJ, 479, 933 Kreykenbohm, I., Wilms, J., Coburn, W., et al. 2004, A\&A, 427, 975

Kreykenbohm, I., et al. 2004, Proceedings of the 5th INTEGRAL Workshop on the INTEGRAL Universe (ESA SP-552), 16-20 February 2004, Munich, Germany, ed. V. Schönfelder, G. Lichti, \& C. Winkler, 333 [arXiv: astro-ph/0406683]

Romero, G. E., Kaufman Bernado, M. M., Combi, J. A., \& Torres, D. F. 2001, A\&A, 376, 599

Schönfelder, V., Bennett, K., Blom, J. J., et al. 2002, A\&AS, 143, 145

Soldi, S., Beckmann, V., Bassani, L., et al. 2005, A\&A, 444, 431

Torres, D. F., Romero, G. E., Combi, J. A., et al. 2001, A\&A, 370, 468

Walter, R., Rodriguez, J., Foschini, L., et al. 2003, A\&A, 411, 427

Zhang, S., Collmar, W., \& Schönfelder, V. 2002, A\&A, 396, 923 